\title{
A Ecocardiografia com Contraste no Diagnóstico de Dilatações Vasculares Intrapulmonares em Candidatos ao Transplante Hepático
}

\author{
Paulo Roberto Pavarino, Hélio Augusto dos Reis Corbucci, Carlos Henrique de Marchi, \\ Paula Fernanda da Mata, Moacir Fernandes de Godoy \\ São José do Rio Preto, SP
}

\section{Objetivo}

Determinar a importância da ecocardiografia com contraste no diagnóstico de dilatações vasculares intrapulmonares em portadores de doenças hepáticas graves, candidatos a transplante.

\section{Métodos}

Estudados 76 pacientes com doenças hepáticas crônicas, sem evidências de doenças intrínsecas pulmonares, insuficiência cardíaca ou cardiopatias congênitas com comunicações intracardíacas, submetidos ao ecocardiograma transtorácico, sendo que em 32 realizou-se, consecutivamente, o estudo transesofágico. 0 resultado da ecocardiografia com contraste foi positivo quando detectada a presença de contraste em câmaras cardíacas esquerdas, 4 a 6 ciclos cardíacos, após opacificação das câmaras cardíacas direitas.

\section{Resultados}

A prevalência de dilatações vasculares intrapulmonares foi de $53,9 \%$ (41/76 pacientes). A sensibilidade, especificidade, valor preditivo positivo, valor preditivo negativo e acurácia do ecocardiograma transtorácico em relação ao transesofágico, na confirmação de anormalidades vasculares pulmonares em hepatopatas foi, respectivamente, de $75 \%, 100 \%, 100 \%, 80 \%$ e $87,5 \%$. 0 grau de oxigenação arterial não apresentou qualquer correlação com a ocorrência de estudo ecocardiográfico positivo.

\section{Conclusão}

A ecocardiografia com contraste mostrou-se eficaz, de utilização fácil e segura em candidatos ao transplante hepático. 0 ecocardiograma transtorácico pode ser empregado na rotina diagnóstica das dilatações vasculares intrapulmonares, reservando-se o estudo transesofágico para os casos inconclusivos com suspeita clínica.

\section{Palavras-chave}

ecocardiografia com contraste, dilatações vasculares intrapulmonares, transplante hepático

Hospital de Base de São J osé do Rio Preto - SP e Faculdade de Medicina de São J osé do Rio Preto - SP

Endereço para Correspondência: Dr. Paulo Roberto Pavarino

Rua J osé Felipe Antonio, 303/32 - Bl.07 - Jd. Vivendas

São J osé do Rio Preto - Cep 15090-430

E-mail: pavarino@cardiol.br

Recebido para publicação em 10/3/03

Aceito em 20/10/03
A associação entre doença hepática e dilatações vasculares pulmonares vem sendo enfatizada por diversos autores há vários anos, permitindo conhecer aspectos fisiopatológicos da hipoxemia arterial encontrada em alguns pacientes com doença hepática crônica, denominada síndrome hepatopulmonar. Esta condição clínica é caracterizada pela tríade de disfunção hepática, dilatação vascular intrapulmonar e hipoxemia ${ }^{1-11}$. A vasodilatação capilar pulmonar é complicação extra-hepática de doença hepática grave, provavelmente por mediação vasoativa do óxido nítrico $0^{2,7,11-13}$, promovendo a ocorrência de shunt direita-esquerda intrapulmonar, com conseqüente alteração na difusão alveolo-capilar e desequilíbrio ventilação/ perfusão pulmonar. 19,11,14,15 Em estágios avançados da doença hepática, tanto a vasodilatação arterial, como as comunicações arteriovenosas pulmonares verdadeiras podem estar presentes ${ }^{1,15}$. Os pacientes podem apresentar gasometria arterial normal ou hipoxemia arterial em $9-29 \%$ dos casos, que pode ser grave e estar associada à cianose e dispnéia. 1,3,7,16,17 Condições hemodinâmicas com débito cardíaco elevado, resistências vasculares sistêmica e pulmonar baixas, e redução do conteúdo de oxigênio arterial e venoso misto, podem, também, estar presentes ${ }^{1,5-7,10-12,18}$.

Dentre os métodos diagnósticos utilizados para identificação de alterações vasculares intrapulmonares em pacientes com doenças hepáticas crônicas, incluem-se a ecocardiografia com contraste, a cintilografia de perfusão pulmonar com macroagregado de albumina marcado com tecnécio99 e a angiografia pulmonar. A ecocardiografia com contraste é considerada padrão-ouro no diagnóstico desta condição, apresentando inúmeras vantagens sobre outros métodos, permitindo, inclusive, detectar shunts intrapulmonares em pacientes com estudo angiográfico e/ou gasometria arterial normais. Estudos recentes destacam a superioridade diagnóstica da ecocardiografia transesofágica com contraste na pesquisa de alterações vasculares pulmonares neste grupo de pacientes $3,6,12,16-19-26$.

O objetivo deste estudo foi comparar os resultados da ecocardiografia com contraste, nas modalidades transtorácica e transesofágica, além de determinar sua importância no diagnóstico de dilatações vasculares intrapulmonares em candidatos ao transplante hepático.

\section{Métodos}

A ecocardiografia com contraste foi realizada, consecutivamente, em 76 pacientes que apresentavam doenças hepáticas graves e 
avançadas, incluídos no protocolo de transplante hepático. Foram excluídos os pacientes com diagnóstico de doenças pulmonares crônicas, insuficiência cardíaca e cardiopatias congênitas com comunicações intracardíacas. 0 estudo foi realizado após esclarecimento individual sobre os objetivos da investigação e consentimento escrito, pós-informado. 0 protocolo de pesquisa foi avaliado e aprovado pelo Comitê de Ética em Pesquisa da Instituição.

A idade média foi de $44 \pm 14,6$ anos, sendo $59(77,6 \%)$ homens e $18(22,4 \%)$ mulheres. Dos 76 pacientes com hepatopatia avançada, 72 apresentavam diagnóstico de cirrose hepática e 4 de fibrose hepática. Entre os pacientes com lesão hepatocelular, 12 apresentavam cirrose alcoólica, 9 hepatite $B, 16$ hepatite $C$, 3 hepatite B e C, 15 cirrose mista (hepatite B e/ou C associada ao álcool), 9 cirrose criptogênica, 2 cirrose auto-imune, 3 cirrose biliar (obstrução biliar primária), 1 hemocromatose, 1 esteatohepatite não alcoólica e 1 com doença de Wilson. Dos pacientes com fibrose intra-hepática ou extra-hepática, 1 apresentava esquistossomose, 1 paracoccidiodomicose e 2 doenças veno-oclusivas (1 trombose de veia porta, 1 síndrome de Budd-Chiari).

Foram utilizados equipamentos da marca ATL (Advanced Technology Laboratories Inc.,B othel, USA) para obtenção de imagens bidimensionais, de acordo com técnicas e cortes previamente estabelecidos, com o paciente em decúbito lateral esquerdo ${ }^{27}$. A ecocardiografia transtorácica com contraste foi realizada em aparelho HDI 5000 com transdutor phased array eletrônico de banda larga, com freqüência de 2 a 4 MHz, utilizando-se a imagem em segunda harmônica em todos os exames, a fim de reduzir artefatos de imagem e aumentar a resolução do contraste. A ecocardiografia transesofágica com contraste foi realizada em aparelho Apogee CX 200, com introdução de sonda esofágica multiplanar de 5,0 $\mathrm{MHz}$ a uma profundidade de aproximadamente $30 \mathrm{~cm}$ da arcada dentária superior, após anestesia local da orofaringe. A utilização do corte ecocardiográfico de 4 câmaras permitiu a visibilização simultânea dos átrios e, quando possível, das veias pulmonares superiores esquerda e direita ${ }^{28,29}$.

Foram realizadas medidas do diâmetro do átrio esquerdo, dimensões diastólica e sistólica do ventrículo esquerdo, obtenção da fração de ejeção do ventrículo esquerdo pelo método de cubagem dos diâmetros e estimativa da pressão sistólica do ventrículo direito pela regurgitação tricúspide, utilizando a equação modificada de Bernoulli28,30-33.

O estudo ecocardiográfico foi desenvolvido seguindo os métodos publicados por Krowka e cols. ${ }^{11}$ e Aller e cols ${ }^{3}$. As microbolhas foram produzidas manualmente, promovendo-se a transferência de $10 \mathrm{~mL}$ de solução salina entre 2 seringas conectadas em equipo de três vias, por 10 a 15 vezes, e, a seguir, administrada rapidamente em acesso venoso periférico. 0 estudo foi considerado positivo, quando detectada a presença anormal de contraste em câmaras cardíacas esquerdas, com um atraso de 4 a 6 ciclos cardíacos, após opacificação inicial das câmaras cardíacas direitas (fig. 1). Em geral foram feitas três injeções para determinar a reprodutibilidade; os resultados foram gravados em fitas de videocassete e analisados por dois observadores. As injeções subseqüentes foram iniciadas somente após completa remoção das microbolhas das cavidades direita e esquerda. De acordo com a opacificação do átrio esquerdo, realizou-se análise semiquantitativa das microbolhas, seguindo os critérios estabelecidos por Aller e 516 cols $^{3}$. A comparação simultânea da intensidade máxima das ima-

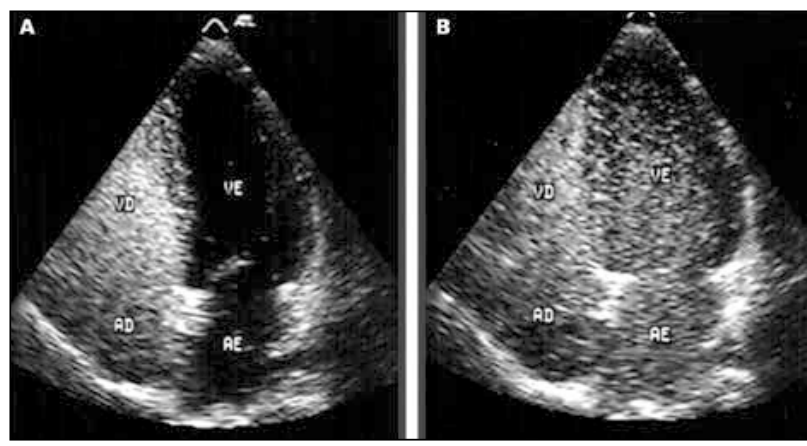

Fig. 1 - Ecocardiograma bidimensional transtorácico, corte apical 4 câmaras: A) presença do contraste em câmaras direitas; B) presença do contraste em câmaras esquerdas após 6 ciclos cardíacos, compatível com dilatações vasculares intrapulmonares

gens ecocardiográficas produzidas pelas microbolhas entre as cavidades cardíacas direita e esquerda, permitiu considerar como grau 1 a ausência de microbolhas; grau 2 a passagem de poucas microbolhas isoladas; grau 3 a passagem de numerosas microboIhas isoladas; grau 4 a passagem de numerosas microbolhas resultando em aumento da ecogenicidade; grau 5 a opacificação do átrio esquerdo, mas em grau menor que 0 átrio direito; grau 6 a opacificação completa do átrio esquerdo similar ao átrio direito. Os graus 1 e 2 foram considerados normais ou ausência de vasodilatações pulmonares; o grau 3, como presença de discretas vasodilatações pulmonares, e finalmente, os graus 4 a 6 como vasodilatações pulmonares significativas ou importantes.

A pressão parcial de oxigênio $\left(\mathrm{PaO}_{2}\right)$ foi determinada em amostras de sangue arterial, colhidas da artéria radial, em ar ambiente, o mais próximo possível do dia do estudo ecocardiográfico (1-3 dias). 0 valor da $\mathrm{PaO}_{2}$, quando inferior a $70 \mathrm{mmHg}$, foi considerado indicativo de hipoxemia arterial.

As variáveis quantitativas contínuas foram analisadas com auxílio do teste $t$ de Student empregando-se a correção de Tukey, caso necessário. A comparação das freqüências foi realizada com auxílio do teste do qui-quadrado ou teste exato de Fisher. Admitiu-se erro alfa de $5 \%$, sendo considerados significantes os valores de $P \leq 0,05$.

\section{Resultados}

Todos os procedimentos diagnósticos foram bem tolerados, e o ecocardiograma transesofágico realizado sem complicações. A utilização da ecocardiografia com contraste, transtorácica e transesofágica, permitiu demonstrar a presença de dilatações vasculares pulmonares em 53,9\% (41/76) dos pacientes. No estudo transtorácico, isoladamente, observou-se uma prevalência de dilatações vasculares intrapulmonares em 48,7\% (37/76) dos casos. Dos 32 pacientes submetidos, consecutivamente, ao estudo transesofágico, 16 apresentaram ecocardiograma com contraste positivo (50\%); ( $P=1,0$; teste exato de Fisher). Quatro pacientes com estudo transtorácico, inicialmente inconclusivo, foram submetidos ao ecocardiograma transesofágico e considerados positivos. A síndrome hepatopulmonar esteve presente em apenas 4 $(5,3 \%)$ pacientes. Seguindo os critérios estabelecidos por Aller e cols. $^{3}, 16$ (21\%) pacientes apresentavam vasodilatações pulmonares discretas; 25 (33\%) vasodilatações pulmonares significantes e os 35 (46\%) estudo ecocardiográfico normal. O ecocardiograma 
transtorácico mostrou sensibilidade de $75 \%$, especificidade de $100 \%$, valor preditivo positivo de $100 \%$, valor preditivo negativo de $80 \%$ e acurácia de $87,5 \%$, no diagnóstico de dilatações vasculares intrapulmonares, quando comparado ao ecocardiograma transesofágico, considerado padrão ouro.

Analisando-se a etiologia da doença hepática, 28 (46,7\%) dos 60 pacientes com cirrose por destruição hepatocelular, 8 $(88,9 \%)$ de 9 pacientes com cirrose criptogênica, 3 com cirrose biliar e 2 com cirrose por doenças veno-oclusivas apresentaram ecocardiograma positivo. Comparando-se os resultados de pacientes com cirrose criptogênica com dilatações vasculares pulmonares aos pacientes com todas as outras etiologias, pelo teste do $\chi^{2}$ não foi encontrada diferença estatística significativa entre os grupos $(P=0,059)$. Entretanto, ao se comparar a cirrose criptogênica isoladamente, com as que cursam com destruição hepatocelular, a diferença foi estatisticamente significativa ( $P=0,044)$.

As variáveis gasométricas e ecodopplercardiográficas estão apresentadas na tabela I. A hipoxemia arterial esteve presente em $9(15,9 \%)$ dos 76 pacientes, sendo que, apenas $4(44,4 \%)$ apresentaram evidências de dilatações vasculares pulmonares. Dos 67 pacientes sem hipoxemia arterial, 37 (55,2\%) apresentaram ecocardiograma positivo.

\section{Discussão}

As mudanças vasculares pulmonares encontradas em hepatopatas crônicos são vasodilatações disseminadas com diâmetros variando de 15 a $150 \mu \mathrm{m}$, mais notável em nível capilar e próximo às áreas de trocas gasosas. 0 desvio de fluxo sangüíneo pulmonar para os capilares dilatados, evita unidades alveolares funcionantes, comprometendo a relação difusão-perfusão pulmonar, com conseqüente queda da saturação arterial de oxigênio ${ }^{1,5,7,9-11}$.

As anormalidades vasculares intrapulmonares não são detectadas rotineiramente, por sua apresentação incomum na população geral e por apresentar aspectos inespecíficos em exames de rotina ${ }^{34}$. Estudos com ecocardiografia de contraste em pacientes com cirrose hepática grave mostram existência de dilatações vasculares intrapulmonares em 13 a $47 \%$ dos pacientes, mesmo em estudos angiográficos normais ${ }^{1,3,4,7,11,16,17,35,36}$. Em nossso estudo, a identificação de dilatações vasculares pulmonares, utilizando-se a ecocardiografia com contraste, foi possível em 41 dos 76 pacientes estudados, ou seja, 53,9\% dos casos, resultado semeIhante aos da literatura.
Estudos de Vedrinne e cols. ${ }^{24}$ e Aller e cols. ${ }^{3}$ demonstraram a superioridade da ecocardiografia transesofágica com contraste no diagnóstico de dilatações vasculares intrapulmonares em candidatos a transplante hepático $0^{3,24,25}$. A ecocardiografia transesofágica, considerada padrão-ouro para o diagnóstico de dilatações vasculares intrapulmonares, em nosso estudo, permitiu demonstrar a presença desta condição em 50\% (16/32) dos casos. Em quatro pacientes com estudo transtorácico previamente inconclusivo, por janela acústica inadequada, somente foi possível a demonstração de dilatações vasculares intrapulmonares após utilização da ecocardiografia transesofágica. A utilização de imagem em segunda harmônica no ecocardiograma transtorácico, em nosso estudo, contribuiu de forma significativa para obtenção de resultados satisfatórios e semelhantes ao estudo transesofágico. Ao se comparar a proporção de indivíduos com dilatações vasculares pulmonares diagnosticada pelo ecocardiograma transtorácico e transesofágico, pelo teste de Fisher, não houve diferença estatística entre os achados $(P=1)$, mostrando que a eficácia dos dois métodos diagnósticos é equivalente. A comparação dos resultados da ecocardiografia transtorácica e transesofágica com contraste, realizada consecutivamente em 32 pacientes, demonstrou sensibilidade de $75 \%$, especificidade de $100 \%$, valor preditivo positivo de $100 \%$, valor preditivo negativo de $80 \%$ e acurácia de $87,5 \%$, o que valida a ecocardiografia transtorácica com contraste e imagem em segunda harmônica como um teste diagnóstico rápido, seguro, incruento, confiável e barato, no estudo destes pacientes.

A síndrome hepatopulmonar, geralmente relatada em 9 a $29 \%$ dos casos de insuficiência hepática ${ }^{1,3,7,16,17}$, em nosso estudo foi encontrada em 5,3\% (4 casos). No presente estudo, o grau de oxigenação arterial não apresentou qualquer correlação estatística com a ocorrência de ecocardiograma positivo. Estes achados são semelhantes aos de Krowka e cols. ${ }^{18}$, que também não encontraram correlação entre anormalidades vasculares pulmonares e gases sangüíneos, em pacientes com ecocardiograma positivo (13,2\% dos casos) comparado àqueles com estudo normal. Mimidis e cols. ${ }^{4}$ também encontraram gasometria arterial normal em 56 indivíduos cirróticos, sendo que apenas 8 (14,3\%) apresentavam ecocardiograma com contraste positivo. Vedrinne e cols. ${ }^{24}$, entretanto, encontraram hipoxemia em $56 \%$ e $33 \%$ dos pacientes, com shunts intrapulmonares diagnosticados pela ecocardiografia transesofágica e transtorácica, respectivamente. Quanto aos valores da $\mathrm{PaO}_{2}$ média encontrados neste estudo, foram semelhantes nos diferentes graus de opacificação de cavidades cardíacas esquerdas $(P=0,859)$, a

\begin{tabular}{|c|c|c|c|}
\hline & $\begin{array}{c}\text { Ecocardiograma } \\
\text { negativo } \\
\text { (grau } 1+2 \text { ) }\end{array}$ & $\begin{array}{l}\text { Ecocardiograma } \\
\text { positivo } \\
\text { (grau } 3+4+5+6 \text { ) }\end{array}$ & Valor de P \\
\hline $\mathrm{PaO}_{2}$ média $(\mathrm{mmHg})$ & $93,2 \pm 17,8$ & $92,4 \pm 20,3$ & 0,859 \\
\hline $\mathrm{PaO}_{2}^{2}<70 \mathrm{mmHg}$ & $5 / 35$ pacientes & 4/41 pacientes & 0,724 \\
\hline PSVD > $30 \mathrm{mmHg}$ & $12 / 35$ pacientes & 16/41 pacientes & 0,850 \\
\hline Diâmetro médio do AE (mm) & $38,9 \pm 5,4$ & $37,2 \pm 4,4$ & 0,135 \\
\hline Diâmetro médio do VE (mm) & $49,8 \pm 4,4$ & $49,8 \pm 6,0$ & 0,981 \\
\hline $\mathrm{DAE}>40 \mathrm{~mm}$ & 11/35 pacientes & 11/41 pacientes & 0,851 \\
\hline DDVE $>55 \mathrm{~mm}$ & 5/35 pacientes & $6 / 41$ pacientes & 0,965 \\
\hline
\end{tabular}


despeito dos resultados de Hopkins e cols. ${ }^{36}$, em que os valores foram significativamente menores em indivíduos com opacificação maior das cavidades esquerdas $(P<0,01)$. Diante de nossos resultados, é possível afirmar que eventuais anormalidades da oxigenação arterial em pacientes com doença hepática crônica não devem ser consideradas indicadoras de shunts intrapulmonares e, isoladamente, não fazem o diagnóstico desta condição.

Frente a uma provável condição circulatória hiperdinâmica existente em indivíduos com shunts vasculares intrapulmonares, que pudesse ocasionar alterações de diâmetro e volume das cavidades esquerdas ou de pressão no leito vascular pulmonar, o presente estudo não encontrou qualquer correlação entre estas variáveis e o diagnóstico de dilatações vasculares intrapulmonares à ecocardiografia com contraste. Quanto aos achados da etiologia da doença hepática, apesar de interessantes, não apresentam suporte fisiopatológico que permita afirmar que distúrbios vasculares pulmonares sejam mais freqüentes em determinados grupos de pacientes com doença hepática crônica.

Em resumo, a ecocardiografia transtorácica com contraste por microbolhas, com utilização de imagem em segunda harmônica, deve ser recomendada na avaliação de rotina e seguimento evolutivo de pacientes com doenças hepáticas graves, candidatos ao transplante hepático, para identificação de dilatações vasculares intrapulmonares ou diagnóstico de síndrome hepatopulmonar. Os casos inconclusivos, com forte suspeita clínica, devem ser submetidos ao estudo transesofágico. 0 significado clínico destes achados no prognóstico de pacientes com doença hepática terminal aguarda estudos futuros.

\section{Referências}

1. Muller C, Schenk P. Hepatopulmonary syndrome. Review article. Wien Klin Wochenschr 1999; 111: 339-47.

2. Barbosa WF, Kondo M. Alterações vasculares pulmonares na hipertensão porta. Rev Soc Cardiol Estado de São Paulo 2000; 10: 609-20.

3. Aller R, Moya J L, Moreira V, et al. Diagnosis of hepatopulmonary syndrome with contrast transesophageal echocardiography. Advantages over contrast transthoracic echocardiography. Dig Dis Sci 1999; 44: 1243-8.

4. Mimidis KP, Vassilakos PI, Mastorakou, et al. Evaluation of contrast echocardiography and lung perfusion scan in detecting intrapulmonary vascular dilation in normoxemic patients with early liver cirrhosis. Hepatogastroenterology 1998; 45 2303-07.

5. Silva AO, D'Albuquerque LAC. Doenças do Fígado. Vol I. Rio de J aneiro: Revinter, 2001:660-3.

6. Krowka MJ, Cortese DA. Hepatopulmonary syndrome: an evolving perspective in the era of liver transplantation. Hepatology 1990; 11: 138-42

7. Castro M, Krowka MJ . Hepatopulmonary Syndrome: A pulmonary vascular complication of liver disease. Clinics Chest Med 1996; 17: 35-48.

8. El-Gamal M, Stoker J B, Spiers EM, Whitaker W. Cyanosis complicating hepatic cirrhosis. Report of a case due to multiple pulmonary arteriovenous fistulas. Am J Cardiol 1970; 24:490-4.

9. Berthelot P, Walker J G, Sherlock S, Reid L. Arterial changes in the lungs in cirrhosis of the liver-lung spider nevi. N EnglJ Med 1966; 274:291-8.

10. Krowka MJ , Cortese DA. Pulmonary aspects of liver disease and liver transplantation. Clin Chest Med 1989; 10: 593-616.

11. Krowka MJ, Cortese DA. Pulmonary aspects of chronic liver disease and liver transplantation. Mayo Clin Proc 1985; 60: 407-18.

12. Aboussouan LS, Stoller J K. The hepatopulmonary syndrome. Baillière's Best Pract Res Clin Gastroenterol 2000; 14: 1033-48.

13. Oh KS, BenderTM, Bowen A, Ledesma-Medina J. Plain radiographic, nuclear medicine and angiographic observations of hepatogenic pulmonary angiodysplasia. Pediatr Radiol 1993; 13: 111-5.

14. Ciappi G, Chiesa A, Chiandussi L, et al. Study of the causes of the hypoxemia in hepatic cirrhosis. Relative importance of pulmonary and extra-pulmonary shunt. Minerva Med 1966; 57: 3533-6.

15. Krowka MJ, Cortese DA. Hepatopulmonary syndrome - Current concepts in diagnostic and therapeutic considerations. Chest 1994; 105: 1528-37.

16. Hourani J M, Bellamy PE, Tashkin DP, Batra P, Simmons MS. Pulmonary disfunction in advanced liver disease: frequent occurrence of an abnormal diffusing capacity. AmJ Med 1991; 90: 693-700.

17. Auletta M, Oliviero U, lasiuolo L, Scherillo G, Antoniello S. Pulmonary hypertension associated with liver cirrhosis: An echocardiography study. Angiology 2000; 51 : 1013-20.

18. Krowka MJ, Tajik J, Dickson R, Wiesner RH, Cortese DA. Intrapulmonary vascular dilatations (IPVD) in liver transplant candidates. Screening by two-dimensional contrast-enhanced echocardiography. Chest 1990; 97: 1165-70.
19. Cerdeña IL, Ojeda FB, Trujillo DA, et al. Pulmonary arteriovenous fistulas. Diagnosis using contrast echocardiography and advantages of the real-time bidimensional technic. Rev Esp Cardiol 1983; 36: 443-6.

20. Barzilai B, Waggoner AD, Spessert C, Picus D, Goodenberg D. Two-dimensional contrast echocardiography in the detection and follow-up of congenital pulmonary arteriovenous malformations. AmJ Cardiol 1991; 68: 1507-10.

21. Shub C, Tajik AJ, Seward J B, Dines DE. Detecting intrapulmonary right-to-left shunt with contrast echocardiography: observations in a patient with diffuse pulmonary arteriovenous fistulas. Mayo Clinic Proc 1976; 51: 81-4

22. Kuramochi T, Izumi S, Nakayama, et al. Contrast echocardiography detection of arteriovenous shunt in a hypoxemic patient with liver cirrhosis. J Cardiol 1994; 24: $155-60$.

23. Hind CR, Wong CM. Detection of pulmonary arteriovenous fistulae in patient with cirrhosis by contrast 2D echocardiography. Gut 1981; 22: 1042-5.

24. Vedrinne J M, Duperret S, Bozollon T, et al. Comparison of transesophageal and transthoracic contrast echocardiography for detection of an intrapulmonary shunt in liver disease. Chest 1997; 111: 1236-40.

25. Ho WJ, Chu PH, Chiang SY, Chiang CW. Localizing intrapulmonary shunt in hepatopulmonary syndrome by transesophageal echocardiography. J pn HeartJ 1999; 40:369-74.

26. Pilatis ND, J acobs LE, Rerkpattanapipat $P$, et al. Clinical predictors of pulmonary hypertension in patients undergoing liver transplant evaluation. Liver Transpl 2000; 6: 85-91.

27. Tajik AJ , Seward J B, Hagler DJ , Mair DD, Lie JT. Two-dimensional real time ultrasonic imaging of the heart and great vessels. Technique, image orientation, structure identification, and validation. Mayo Clin Proc 1978; 53: 271-303.

28. MorcefFAP. Ecocardiografia Uni-Bidimensional, Transesofágica e Doppler. $2^{a} \stackrel{a}{ }$ ed. Rio deJ aneiro: Revinter, 1996.

29. Assef J E, Belém L, Castro-Lima A, Torreão J AM. Ecocardiografia Transesofágica: Atlas-Texto. Rio de J aneiro: Revinter, 2000

30. Sahn DJ , DeMaria A, Kisslo J, Weyman A. Recommendations regarding quantitation in M-mode echocardiography: results of a survey of echocardiography measurements. Circulation 1978; 58: 1072-83.

31. Pombo J F, Troy BL, Russel RO. Left ventricular volumes and ejection fraction by echocardiography. Circulation 1978; 42: 480-90.

32. Feingenbaum H. Echocardiography. 5th ed. Philadelphia: Lea \& Febiger, 1994.

33. Ortiz J, Silva CES, Ghefter CGM, et al. 0 Ecocardiograma no Apoio à Decisão Clínica. $2^{\text {a }}$ ed. Rio de J aneiro: Revinter; 1997.

34. Gianesella RB, Rossi Filho RI, Zielinsky P. Diagnóstico e terapêutica da fístula arteriovenosa pulmonar na infância. Descrição de caso e revisão da literatura. Arq Bras Cardiol 2001; 77: 274-7

35. Murakami J W, Rosembaum DM. Right-to-left pulmonary shunting in pediatric hepatopulmonary syndrome. Clin Nucl Med 1999; 24: 897.

36. Hopkins WE, Waggoner AD, Barzilai B. Frequency and significance of intrapulmonary right-to-left shunting in end-stage hepatic disease. AmJ Cardiol 1992; 70: 516-9. 